\title{
Extensional and Fractural Properties of Concentrated Solutions of Monodisperse Polystyrene at Elevated Temperatures
}

\author{
Eiichi KAMEI* and Shigeharu ONOGI** \\ Department of Polymer Chemistry, Kyoto University, Kyoto, 606 Japan.
}

(Received January 12, 1976)

\begin{abstract}
The extensional and fractural properties were measured for concentrated solutions of polystyrene over wide ranges of extension rate and temperature. From stress-strain curves failure parameters such as failure stress $\sigma_{\mathrm{f}}$ and failure strain $\gamma_{\mathrm{f}}$, fractural parameters such as breaking stress $\sigma_{\mathrm{b}}$ and breaking strain $\gamma_{\mathrm{b}}$, and extensional parameters such as time dependent maximum extensibility $n(t)^{1 / 2}$ and extensional modulus $E(t)$ were evaluated and compared with those of bulk polystyrenes. The dependence of these parameters on the concentration was also discussed. The height of the rubbery plateau, $E_{\mathrm{eN}}^{\circ}$, in the time curves of the elongational modulus is proportional to $\left(\rho_{\mathrm{s}} w\right)^{-2}$, where $\rho_{\mathrm{s}}$ is the density of solution and $w$ is the weight fraction of polymer in solution. This result is consistent with the previous results of our dynamic viscoelastic measurements for polystyrene solutions. The master curves of $n(t)^{1 / 2}$ show a concentration dependence in the plateau region and are similar in shape to the master curves of the draw ratio at the breaking point, $\lambda_{b}$, in the short time scale region below the maximum $\lambda_{b}$. This result indicates that the master curve of $\lambda_{b}$ on the short time scale side below the maximum point may obey the stored energy constant criterion for fracture. The variation of the $\gamma_{b}$ with time depends upon polymer concentration and also shows a much sharper maximum compared with that for the bulk polystyrenes in the intermediate rate region. Such a very sharp maximum is characteristic of solutions.
\end{abstract}

The concentration dependence of the failure envelope, the average molecular weight $M_{\mathrm{e}}$ and the chain length $Z_{\mathrm{e}}$ between entanglement loci were also discussed.

KEY WORDS Extension / Fracture / Concentrated Solution

Monodisperse Polystyrene / Stress-Strain Curve /

In our previous papers, ${ }^{1,2}$ extensional and fractural properties of bulk polystyrenes were measured at several elevated temperatures and rates of strain, and the effects of various factors such as temperature, extension rate, molecular weight, and molecular weight distribution were studied. The main results obtained are summarized as follows:

1. An isochronal stress-strain curve below the failure point can be expressed by the modified Treloar equation for rubber elasticity., ${ }^{3,19,20}$

2. The fractural and failure behaviors can be distinguished into ductile, ductile to flow

* Present address: Hirakata Plastic Laboratory, Ube Industries, Ltd., Hirakata, Osaka, 573 Japan.

** To whom all communications should be addressed. transition, and flow regions. The effect of molecular weight on these behaviors is notable especially in the ductile to flow transition and flow regions.

3. Logarithmic plots of the time required for a specimen to reach its failure point against the root mean square of the second invariant of the strain rate tensor between $t=0$ and $t=t$ are similar in shape to those of the shear viscosity plotted against rate of shear. The slope of the linear part of the curves is in close agreement with that predicted by the viscosity theory of Graessley. ${ }^{4,5}$

These results suggest that the fractural and failure phenomena are closely related to the elongation and the slippage of molecular chains between entanglement loci. 
Many investigations ${ }^{6-15}$ of the effects of polymer concentration on the rheological properties of amorphous polymers have revealed that the entanglement spacing is strongly affected by the polymer concentration. Also, it has been emphasized that the rheological studies on concentrated polymer solutions are very important to clarify the effect of entanglement coupling on the viscoelastic properties of high polymers. ${ }^{16-18}$

The objective of this paper is to study the effect of concentration, if any, on the extensional and fractural properties of narrow-distribution or monodisperse polystyrene.

\section{EXPERIMENTAL}

\section{Materials}

Narrow-distribution polystyrene prepared by anionic polymerization was employed in this study.

The characterization of the polystyrene sample was carried out by the same method as has been described in previous papers. ${ }^{1,2}$ The weightaverage molecular weight $M_{w}$, number-average molecular weight $M_{n}$, and their ratio $\left(M_{w} / M_{n}\right)$ for the bulk sample (LK) are $6.72 \times 10^{5}, 5.87 \times 10^{5}$, and 1.14 , respectively. As is evident from the value of $\left(M_{w} / M_{n}\right)$, the molecular weight distribution is very narrow.

Kaneclor 600 (KC6) was used as the solvent; this is a partially chlorinated biphenyl produced by Kanegafuchi Chemical Industry Co. To prepare concentrated solutions, the required amounts of polymer and solvent (KC6) were dissolved in a large amount (several times in weight) of dichloromethane under stirring, and then dichloromethane was completely removed by evaporation from the mixture in a vacuum oven at $60^{\circ} \mathrm{C}$. The sample codes and polymer concentrations $w$ in weight fraction are tabulated in Table I, where $S$ denotes solution of the bulk sample LK. The last two numbers of the sample codes denote the weight percent of polymer in solution.

Sample films were prepared by melt-pressing of solutions in a laboratory press. The thickness of the films thus prepared was about $0.5 \mathrm{~mm}$. Rectangular specimens, $2 \mathrm{~cm}$ in length and 0.5 $\mathrm{cm}$ in width, were cut from the films and subjected to stress-strain tests.
Table I. Sample codes and weight fractions of polystyrene solutions in $\mathrm{KC} 6$

\begin{tabular}{cc}
\hline Sample & $W$ \\
\hline LK & 1.00 \\
S 90 & 0.90 \\
S 70 & 0.70 \\
S 60 & 0.60 \\
S 50 & 0.50 \\
\hline
\end{tabular}

\section{Measurements}

Stress-strain tests were carried out by means of an Instron-type tensile tester, Tensilon UTM 4L. In order to keep the temperature constant, an oven specially designed by us was employed; this has been described elsewhere. ${ }^{2}$

The initial length $L_{0}$ of the specimen was adjusted to be $1 \mathrm{~cm}$, and measuring temperatures ranged from 56.7 to $176.7^{\circ} \mathrm{C}$. The tests were carried out at seven cross head speeds, $v$, ranging from $5 / 6$ to $0.5 / 60 \mathrm{~cm} / \mathrm{sec}$.

The specimen length $L$ varies with time $t$ according to

$$
L=L_{0}+v t \text {. }
$$

Therefore, the extension ratio $\lambda$ is given by

$$
\lambda=\frac{L}{L_{0}}=\frac{L_{0}+v t}{L_{0}} .
$$

The true strain $\gamma$ is defined as

$$
\gamma=\int_{0}^{t} \dot{\gamma} \mathrm{d} t=\int_{0}^{t} \frac{v}{L_{0}+v t} \mathrm{~d} t=\ln \lambda,
$$

where $\dot{\gamma}$ is the rate of strain given by

$$
\dot{\gamma}=\frac{v}{L}=\frac{v}{L_{0}+v t} .
$$

The rate of strain tensor $\Delta$ can be expressed as

$$
\Delta=\frac{v}{L_{0}}\left(\begin{array}{ccc}
2 / \lambda & 0 & 0 \\
0 & -1 / \lambda & 0 \\
0 & 0 & -1 / \lambda
\end{array}\right) .
$$

Then, the second invariant of the rate of strain tensor is given by

$$
\Pi_{\Delta}=8\left(\frac{v}{L_{0}}\right)^{2}\left(\frac{1}{\lambda}\right)^{2} .
$$

Therefore, the average rate of strain between $t=0$ and $t=t$ is given by

$$
\left\langle\Pi_{\Delta}{ }^{1 / 2}\right\rangle=\frac{1}{t} \int_{0}^{t} \Pi_{\Delta}{ }^{1 / 2} \mathrm{~d} t=\frac{\sqrt{8}\left(v / L_{0}\right)}{\lambda-1} \ln \lambda .
$$


The procedures for evaluating the extensional properties, including the above quantities, of bulk polystyrenes reported in a previous paper $^{2}$ were also used for the concentrated polystyrene solutions in this study, because the stressstrain behavior of concentrated polystyrene solutions is very similar to that of bulk polystyrenes. Equations 8 and 9 obtained previously ${ }^{2}$ were adopted to describe the length of the sample below and above the failure point. Below the failure point, the actual elongation is 1.155 times larger than the apparent elongation simply obtained from the separation of the jaws. Therefore, the actual elongation $L$ is given by

$$
L=L_{0}+1.155 \mathrm{vt} \text {. }
$$

Beyond the failure point, specimens prepared from concentrated solutions were elongated mostly in their centers, as was the case of the bulk specimens in the previous study, ${ }^{2}$ and the actual elongation became much larger than the apparent elongation. Moreover, the stress $\sigma$ decreased very rapidly. Hereafter, the failure point will be denoted by the subscript $f$. Some specimens, however, elongate further beyond the failure point, until they break completely at a certain point. This breaking point will be denoted by the subscript $b$.

The specimen length $L^{\prime}$ beyond the failure point can be expressed by

$$
L^{\prime}=\left(L_{0}+1.155 v t_{\mathrm{f}}\right) \exp \left[\dot{\gamma}_{\mathrm{f}}\left(t^{\prime}-t_{\mathrm{f}}\right)\right], \quad t_{\mathrm{f}}<t^{\prime} \leq t_{\mathrm{b}}
$$

where $t_{\mathrm{f}}$ and $t_{\mathrm{b}}$ are the times required for the specimen to reach its failure and breaking points, respectively. $\dot{\gamma}_{\mathrm{f}}$ is the rate of strain at the failure point. Then, the extension ratio $\lambda$ at any arbitrary time $t$ is given by

or

$$
\lambda=\frac{L}{L_{0}}, \quad \text { (below failure point) }
$$

$$
\lambda=\frac{L^{\prime}}{L_{0}} . \quad \text { (above failure point) }
$$

The true stress $\sigma$ was determined as the product of the nominal (or engineering) stress $\sigma_{\mathrm{a}}$ and $\lambda$. All data for the specimens which were broken near the clamps were excluded.

\section{RESULTS AND DISCUSSION}

\section{Stress-Strain Curves}

The stress-strain behavior of concentrated polystyrene solutions depends not only upon temperature and extension rate, as in the case of bulk polystyrene, ${ }^{1,2}$ but also upon polymer concentration. Figure 1 shows a typical example of stress-strain curves, obtained at six different temperatures ranging from 75.3 to $134.5^{\circ} \mathrm{C}$ at a constant extension rate of $5 \mathrm{~cm} / \mathrm{min}$. Figure 1a shows $\sigma_{\mathrm{a}} v s .(\lambda-1)$ curves for $\mathrm{S} 70$, whose con-

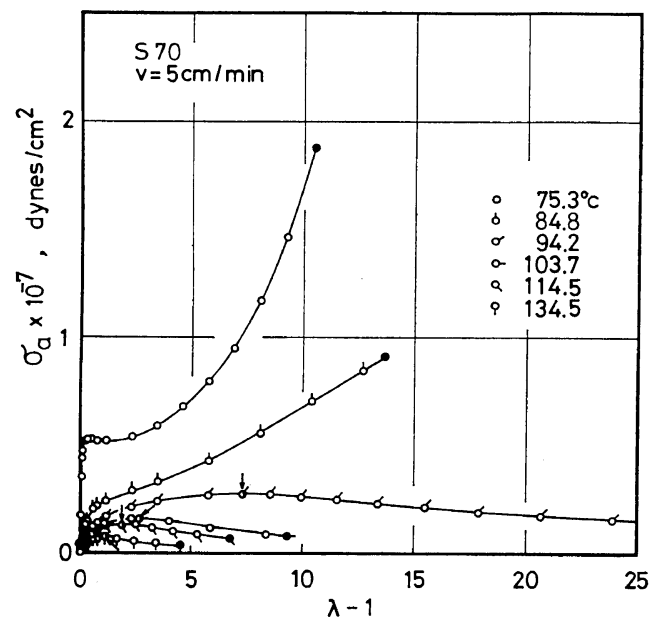

(a)

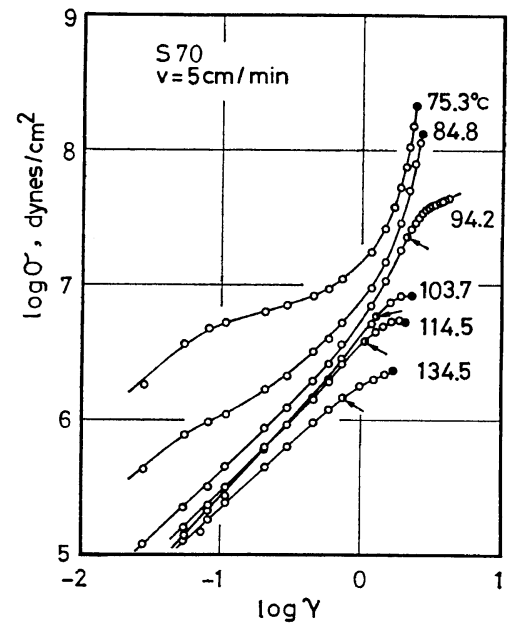

(b)

Figure 1. A typical example of stress-strain curves obtained for S70 at six different temperatures and at a constant extension rate of $5 \mathrm{~cm} / \mathrm{min}$. 
centration is $70 \mathrm{wt} \%$, while Figure $1 \mathrm{~b}$ shows $\log \sigma$ vs. $\log \gamma$ curves for the same specimen. In these figures, the semiclosed circles pointed by arrows indicate the failure point and the closed circles indicate breaking points. As is evident from these figures, the level and shape of the stress-strain curves depend strongly upon temperature, and $\log \sigma$ vs. $\log \gamma$ curves can be approximated by straight lines having a slope of about unity in the small strain region. The stress-strain curves below $84.8^{\circ} \mathrm{C}$ are different in shape from those above $94.2^{\circ} \mathrm{C}$. As was pointed out in a previous paper, ${ }^{2}$ the change in the shape of the stress-strain curves below $84.8^{\circ} \mathrm{C}$ results primarily from a decrease of the maximum extensibility, ${ }^{19-24}$ while the change in the shape above $94.2^{\circ} \mathrm{C}$ results from a counterbalance of two competing processes: (1) the increase in stress due to the extension of molecular chains between entanglement loci, and (2) the relief of stress due to the slippage of polymer chains at entanglement loci.

Figure 2 shows that the effect of extension rate on stress-strain behavior is similar to that of temperature, as seen in Figure 1, owing to the role of the friction coefficient, or local viscous resistance. ${ }^{19-24}$ Here again, semiclosed and closed circles indicate the failure and breaking points, respectively. As is evident in Figure $2 b$, the final steep portion of the curves before breaking shifts progressively to the left with increasing extension rate. This change results from the time-dependent nature of the maximum extensibility. ${ }^{19-22}$

Many investigations $s^{6,7,18,25}$ of the effect of concentration on the rheological properties of amorphous polymers have revealed that the free volume increases with decreasing concentration. Therefore, glass transition temperatures decrease and materials become softer as the concentration decreases. ${ }^{18}$ It is anticipated from these results that the effect of concentration on the extensional behavior will be very similar to that of temperature and extensional rate. Figure 3 includes $\sigma_{\mathrm{a}} v s .(\lambda-1)$ (a) and $\log \sigma v s . \log \gamma$ (b) curves for four samples at different concentrations extended at a constant extension rate of $5 \mathrm{~cm} / \mathrm{min}$ at $103.7^{\circ} \mathrm{C}$. It is apparent from these figures that the effect of concentration is very marked over the entire range of strain. Stress at a given strain and maximum stress increase with increasing concentration. Comparing this figure with Figures 1 and 2, it is obvious that the effect of concentration is similar to those of extensional rate and temperature. As described in previous papers, ${ }^{1,2}$ bulk polystyrene behaves in a brittle manner at this temperature and extension rate, because the temperature is near the glass transition temperature $\left(100^{\circ} \mathrm{C}\right)$ for bulk polystyrene. In contrast to bulk polystyrene,

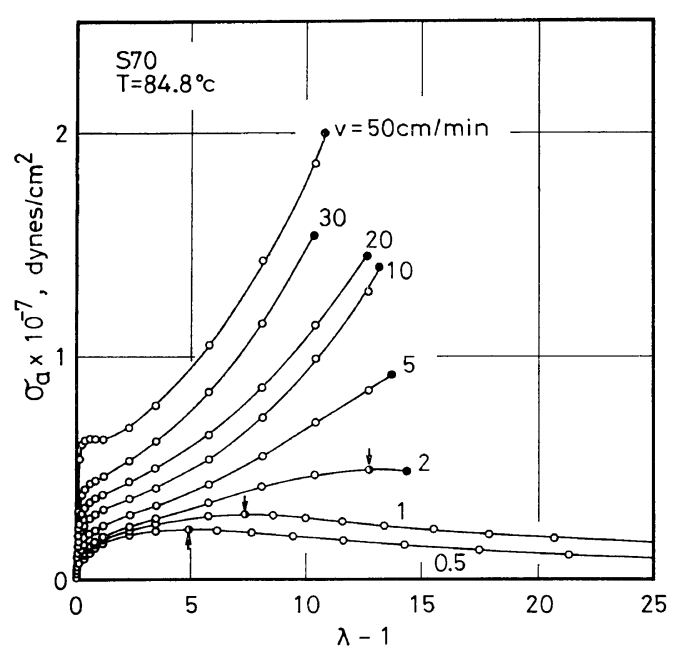

(a)

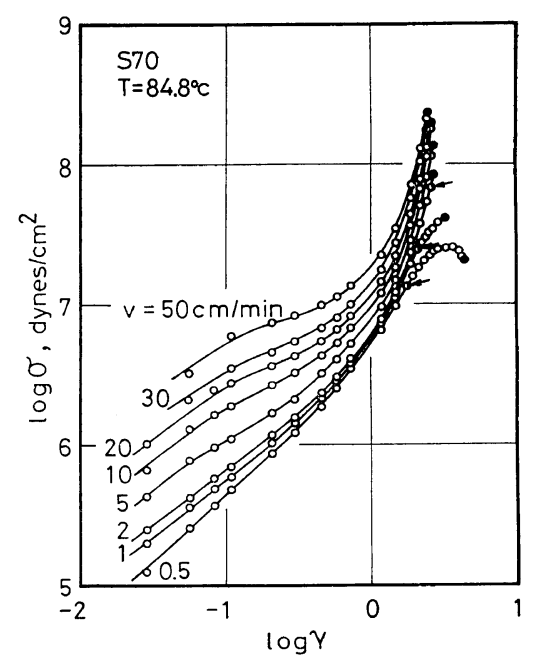

(b)

Figure 2. Stress-strain curves obtained for $S 70$ at $84.8^{\circ} \mathrm{C}$ at seven different extension rates. 


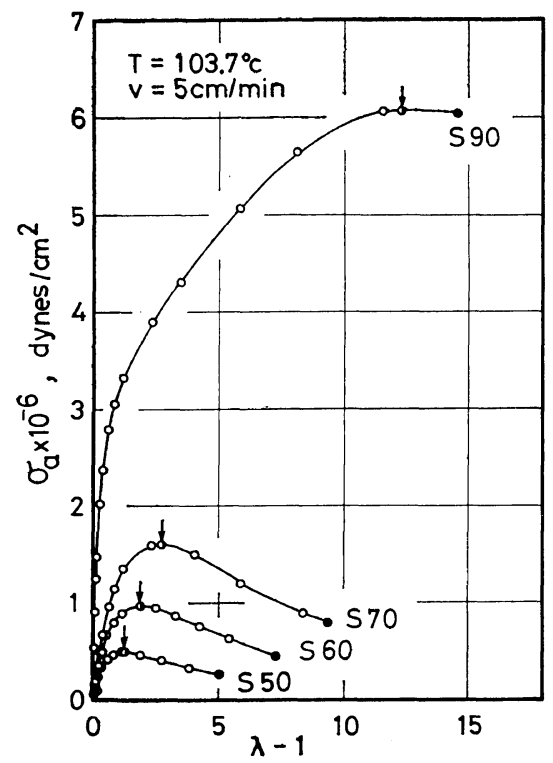

(a)

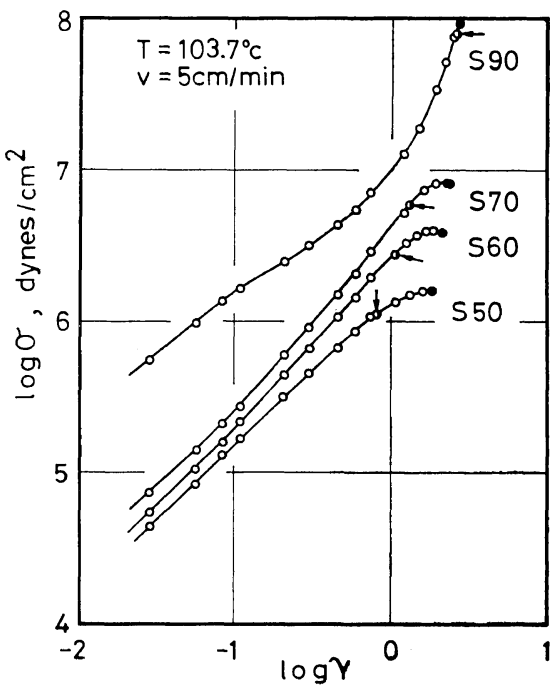

(b)

Figure 3. Stress-strain curves for four samples at different concentrations extended at a constant extension rate of $5 \mathrm{~cm} / \mathrm{min}$ at $103.7^{\circ} \mathrm{C}$.

all the solutions give ductile elongational and fractural behaviors at the same temperature and extension rate. The difference arises from the decrease in glass transition temperature with decreasing concentration.

\section{Modulus of Elasticity and Isochronal Stress- Strain Curves}

As mentioned above, the apparent stressstrain curves for polystyrene solutions also show the failure point below the breaking point at temperatures higher than glass transition temperatures or at lower extension rates, as in the case of bulk polystyrenes. ${ }^{2}$ Beyond the failure point, the strain in the specimen is localized inhomogeneously from place to place; the specimen is elongated at its center. As is evident from our previous study, ${ }^{2}$ when strain increases according to eq 8 below the failure point, the irrecoverable strain of the specimen can be ignored. This result indicates that the specimen behaves like vulcanized rubber in this region of strain. On the other hand, when the strain increases according to eq 9 beyond the failure point, the irrecoverable strain increases progressively with increasing strain, indicating that some slippage of molecular chains at entangle- ment loci occurs. Therefore, this region of strain is characteristic of uncrosslinked amorphous polymers elongated at higher temperatures or at lower extension rates. From the resemblance of the stress-strain behavior for concentrated polystyrene solutions to that for bulk polystyrene, it is expected that isochronal stressstrain curves for solutions below the failure point may also be expressed by the following modified Treloar equation for the rubber elasticity, ${ }^{3,19,20}$ as shown for bulk polymers in a previous paper: ${ }^{2}$

$$
\sigma(\lambda, t)=\frac{E(t)}{3} \lambda\left[\frac{n(t)^{1 / 2}}{3} \mathscr{L}^{-1}\left(\frac{\lambda}{n(t)^{1 / 2}}\right)-\frac{1}{\lambda^{2}}\right]
$$

where $E(t)$ is the time dependent Young's modulus, $n(t)$ is the number of time-dependent statistical random links in molecular chains between entanglement loci, and $\mathscr{L}^{-1}(x)$ is the inverse Langevin function. When $\lambda$ is equal to $\left.n^{(} t\right)^{1 / 2}$, the value of $\sigma(\lambda, t)$ becomes infinity. At very small strains, this equation reduces to

$$
\sigma(\lambda, t)=E(t)(\lambda-1)
$$

Thus, $E(t)$ can be obtained from the linear portion of the isochronal stress-strain curves, 


\section{E. KAMEI and S. ONOGI}

and $n(t)^{1 / 2}$ can be determined from the experimental curves by curve-fitting. It is thus very interesting to investigate how $E(t)$ and $n(t)^{1 / 2}$ for polymer solutions change with time below the failure point.

In Figure 4 isochronal stress-strain curves are shown; these were obtained for $\mathrm{S} 70$ at $84.8^{\circ} \mathrm{C}$ and at five different times between $6 \mathrm{sec}$ and

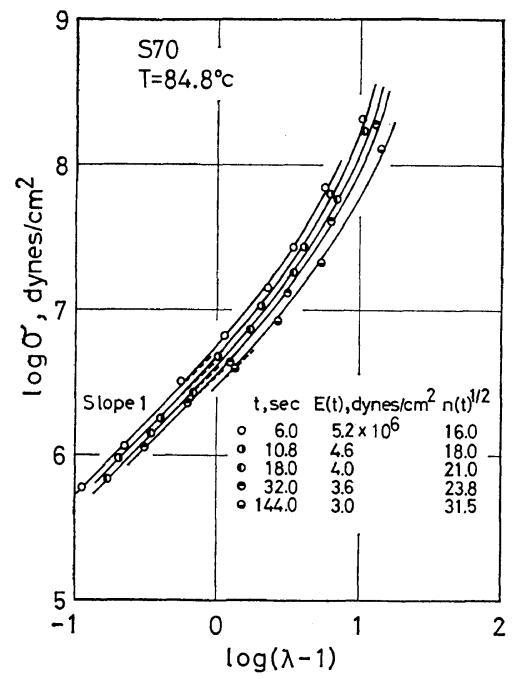

Figure 4. Isochronal stress-strain curves obtained for $\mathbf{S} 70$ for five different times at $84.8^{\circ} \mathrm{C}$.
$144 \mathrm{sec}$. In this figure, the broken lines have a slope of unity. It is evident from this figure that each curve has a linear portion with a slope of unity at small strains. This linear portion enables us to evaluate the elongational modulus. The solid lines in the figure represent the values of $\sigma(\lambda, t)$ calculated from eq 10, assuming the values of $E(t)$ and $n(t)^{1 / 2}$ for each time $t$ as tabulated in this figure. The calculated values coincide very well with the experimental ones (circles). This fact indicates that the extensional behavior of concentrated polystyrene solutions below the failure point is very similar to that of vulcanized rubbers, and that entanglements between polymer molecules have the same effect on mechanical properties as do crosslinks. ${ }^{2}$ Therefore, it can be concluded that the deformation below the failure point is governed mainly by the motion of molecular chains between entanglement loci, while the deformation above the failure point is governed by the slippage of molecular chains.

Figure 5 shows master curves of the modulus $E(t)$ evaluated from the isochronal stress-strain curves, as shown in Figure 4, for concentrated polystyrene solutions of different concentrations in the iso-free-volume state in which the fractional free volume $f$ is equal to 0.0501 . The

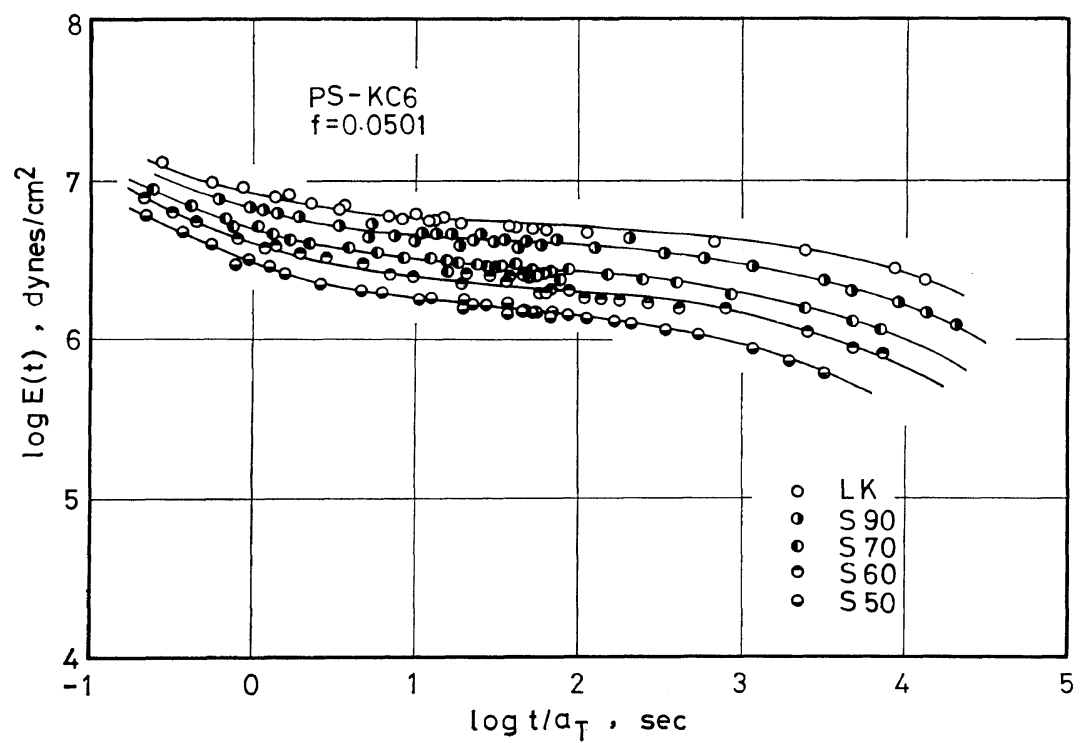

Figure 5. Master curves of $E(t)$ evaluated from the isochronal stress-strain curves, as shown in Figure 4, for bulk polystyrene LK and its solutions at the iso-free-volume state, $f=0.0501$. 
Table II. Entanglement modulus $E_{\mathrm{eN}}^{\circ}$, average molecular weight $M_{\mathrm{e}}$, and chain length $Z_{\mathrm{e}}$ between entanglement loci, modulus $E(t)_{\mathrm{c}}$ and number of time-dependent statistical random links $n(t)_{\mathrm{c}}$ at the critical point in Figure 8, and $n(t)$ obtained from modified failure envelope in Figure 11 for bulk polystyrene LK and its solutions

\begin{tabular}{ccccccc}
\hline Sample & $E_{\mathrm{eN}}^{\circ}$, dyn $/ \mathrm{cm}^{2}$ & $M_{\mathrm{e}}$ & $Z_{\Theta}$ & $E(t)_{\mathrm{c}}, \mathrm{dyn} / \mathrm{cm}^{2}$ & $n(t)_{\mathrm{c}}$ & $n(t)$ \\
\hline LK & $5.6 \times 10^{6}$ & 19000 & 365 & $5.6 \times 10^{6}$ & 729 & 676 \\
S 90 & $4.2 \times 10^{6}$ & 22000 & 423 & $4.5 \times 16^{6}$ & 773 & 729 \\
S 70 & $2.8 \times 10^{6}$ & 26000 & 500 & $3.0 \times 10^{6}$ & 818 & 882 \\
S 60 & $2.1 \times 10^{6}$ & 30000 & 577 & $2.4 \times 10^{6}$ & 949 & 1000 \\
S 50 & $1.7 \times 10^{6}$ & 31000 & 596 & $1.7 \times 10^{6}$ & 1210 & 1180 \\
\hline
\end{tabular}

abscissa of the figure is the reduced time $t / a_{T}$, where $a_{T}$ is the shift factor. The reference temperature and shift factor used in this figure will be discussed later. The master curves range from the end of the transition region to the beginning of the flow region, as can be seen in this figure. The height of the rubbery plateau becomes lower and lower, and the flow region shifts to the short time scale side as the concentration decreases. The curves are very similar to those of the storage shear modulus $G^{\prime}$ obtained for similar polystyrene solutions in previous papers. ${ }^{7,8}$ The height of the rubbery plateau $E_{\mathrm{eN}}^{\circ}$ in the time curves of the elongational modulus is the most important parameter which characterizes the rubbery region; $E_{\mathrm{eN}}^{\circ}$ can be related to the average molecular weight $M_{\mathrm{e}}$ or chain length $Z_{\mathrm{e}}$ between the entanglement loci by

$$
E_{\mathrm{eN}}^{\circ}=3 g_{\mathrm{N}} \rho R T / M_{\Theta}
$$

or

$$
Z_{\mathrm{e}}=j M_{\mathrm{e}} / M_{0}
$$

where $g_{N}$ is a front factor near unity, $\rho$ the density of polymers, $R$ the gas constant, $T$ absolute temperature, $j$ the number of chain atoms per monomer unit, and $M_{0}$ the molecular weight of the monomer unit, which is 104 for polystyrene. In the case of a solution, $\rho$ should be replaced by $\rho_{\mathrm{s}} w$, where $\rho_{\mathrm{s}}$ is the density of the solution. This equation is equivalent to an equation relating the equilibrium modulus to the average molecular weight $M_{\mathrm{c}}$ between crosslinking points in the kinetic theory of rubber elasticity. ${ }^{3}$ The values of $E_{\mathrm{eN}}^{\circ}$ obtained from the height of the rubbery plateaus at the inflection points of the time curves of the elonga- tional modulus are tabulated in Table II, together with those of $M_{\mathrm{e}}$ and $Z_{\mathrm{e}}$ determined from $E_{\mathrm{eN}}^{\circ}$. $E_{\mathrm{eN}}^{\circ}$ decreases gradually as the concentration decreases, while $M_{\mathrm{e}}$ and $Z_{\mathrm{e}}$ increase. The values for the bulk sample LK are in good agreement with those obtained for narrow-distribution polystyrenes in our laboratory, i.e., $E_{\mathrm{eN}}^{\circ}=3 G_{\mathrm{eN}}^{\circ}=$ $5.97 \times 10^{6}, M_{\mathrm{e}}=18100$, and $Z_{\mathrm{e}}=347 .^{7,26}$

The relationship between $E_{\mathrm{eN}}^{\circ}$ and $\rho_{s} w$ is shown in Figure 6, and plots of $M_{\mathrm{e}}$ and $Z_{\mathrm{e}}$ against $\rho_{\mathrm{s}} w$ are given in Figure 7. The plot of $E_{\mathrm{eN}}^{\circ}$ can be approximated by a straight line having the slope of 2, indicating that $E_{\mathrm{eN}}^{\circ}$ is proportional to $\left(\rho_{s} w\right)^{2}$. According to eq 12, the average molecular weight $M_{\mathrm{e}}$ and the chain length $Z_{\theta}$ between entanglement loci should increase in proportion to the reciprocal of $\rho_{s} w$. The plots of $M_{\mathrm{e}}$ and $Z_{\mathrm{e}}$ against $\rho_{s} w$ given in Figure 7 support this. These results are also consistent

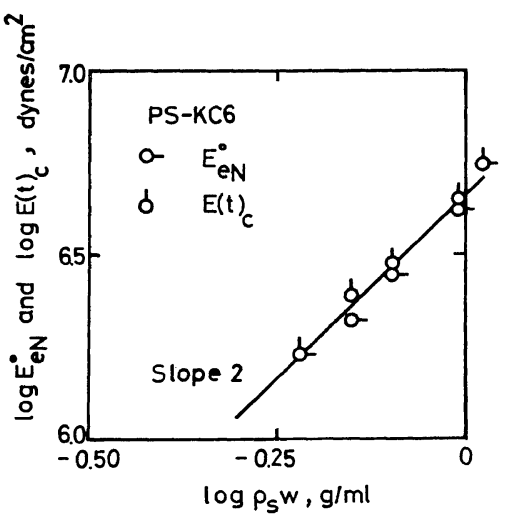

Figure 6. Concentration dependence of quasiequilibrium modulus $E_{\mathrm{eN}}^{\circ}$ obtained from the $E(t)$ curve in Figure 5 and $E(t)_{\mathrm{c}}$ obtained from the critical point in Figure 8 for polystyrene solutions in KC6. 


\section{E. KAMEI and S. ONOGI}

with previous results ${ }^{7}$ of our dynamic viscoelastic measurements for polystyrene solutions.

It can be expected from the definition of $n(t)$ that it is proportional to $M_{\mathrm{e}}$ in the rubbery state. Accordingly, the time dependent Young's modulus $E(t)$ becomes a function of $n(t)^{1 / 2}$. In Figure $8, n(t)^{1 / 2}$ is logarithmically plotted against $E(t)$ for the five samples of different concentrations. Each curve can be approximated by two straight lines, having different slopes of -4 and -1 . The intersection of the straight lines will

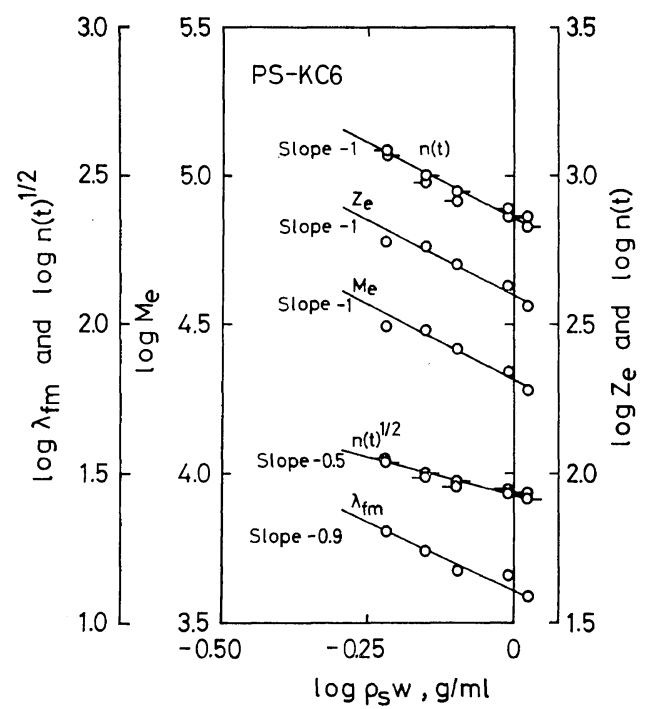

Figure 7. Concentration dependence of $M_{\mathrm{e}}, Z_{\mathrm{e}}$, $n(t), n(t)^{1 / 2}$, and $\lambda_{\mathrm{fm}}$ for polystyrene solutions in KC6: $\quad-, n(t), n(t)^{1 / 2}$ evaluated from $\log \sigma_{\mathrm{f}} / E\left(t_{\mathrm{f}}\right) v s$. $\log (\lambda-1)$ curve in Figure $11 ;-0, n(t)_{\mathrm{c}}, n(t)_{\mathrm{c}} \mathrm{1}^{1 / 2}$ obtained from the critical point in Figure 8.

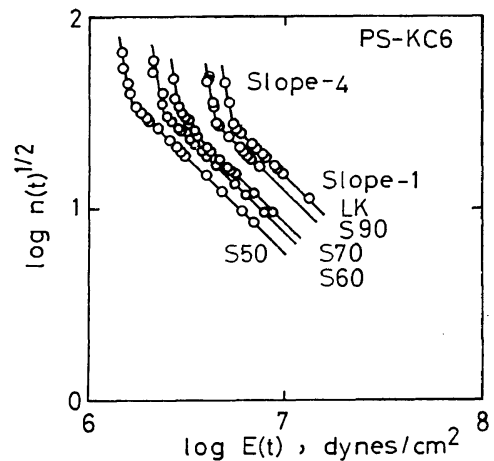

Figure 8. Logarithmic plot of $n(t)^{1 / 2}$ against $E(t)$ for bulk polystyrene LK and its solutions in KC6. be referred to as the "Critical Point" and represented by the subscript c. The curves shift diagonally as concentration increases: the higher the concentration, the higher the $E(t)$ value at a given $n(t)^{1 / 2}$ becomes. The values of $E(t)$ and $n(t)$ at the critical point of the $n(t)^{1 / 2}$ curves, $E(t)_{\mathrm{c}}$ and $n(t)_{\mathrm{c}}$, also vary with concentration, as shown in Table II as well as in Figures 6 and 7. As is evident from Figure $6, E(t)_{\mathrm{c}}$ agrees well with $E_{\mathrm{e} N}^{\circ}$ obtained from the height of the master curve of $E(t)$, and is proportional to $\left(\rho_{\mathrm{s}} w\right)^{-2}$. This result suggests that the critical point just corresponds to the rubbery state. Therefore, it is expected that the values of $n(t)_{\mathbf{c}}$ should be proportional to $M_{e}$ or $Z_{\mathrm{e}}$. As can be seen from Figure $7, n(t), M_{\mathrm{e}}$, and $Z_{\mathrm{e}}$ show the same dependence upon concentration, and $n(t)$ is about two times larger than $Z_{\mathrm{e}}$, indicating that the above condition is fullfilled.

It is well known that $E(t)$ in the transition region is nearly proportional to $t^{-1 / 2} \cdot{ }^{18}$ Since $n(t)$ in Figure 8 decreases in proportion to $E(t)^{2}$ above the critical point, it should increase in proportion to $t$, provided that the region above the critical point corresponds to the transition region. On the other hand, $n(t)$ below the critical point shows a stronger dependence upon $E(t)$ compared with that in the higher $E(t)$ region. This fact means that there exist different molecular mechanisms of deformation in the higher and lower $E(t)$ regions. These mechanisms have different types of relaxation times: ${ }^{18}$ one is associated with the motion of molecular chains

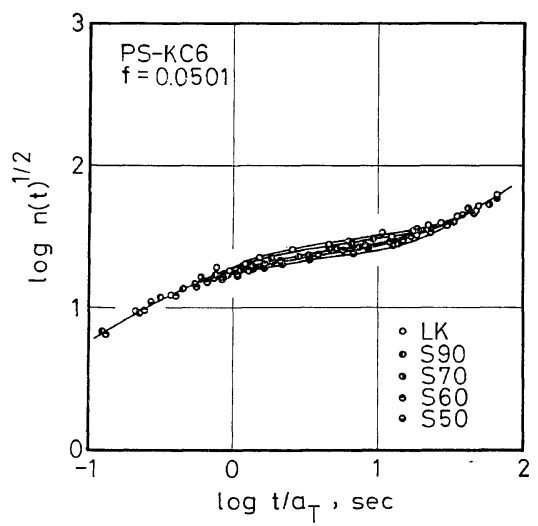

Figure 9. Master curves of $n(t)^{1 / 2}$ for bulk polystyrene LK and its solutions at the iso-free-volume state, $f=0.0501$. 
between entanglement loci, and the other with the slippage of molecular chains.

In Figure 9 master curves of $n(t)^{1 / 2}$ are shown at the iso-free-volume state where $f=0.0501 . n(t)^{1 / 2}$ increases with increasing time, but shows a plateau in the intermediate region. All the curves for all the samples of different concentrations unite with each other at the short and long time scale ends. However, in the plateau region, $n(t)^{1 / 2}$ shows a concentration dependence and increases with increasing concentration, suggesting that $M_{\mathrm{e}}$ should increase with decreasing concentration, as already shown in Figure 7.

\section{Failure Envelope}

Figure 10 gives failure envelopes ${ }^{19}$ for the five samples. On the envelopes, the failure point moves counter-clockwise as the extension rate increases or temperature decreases, until $\lambda_{\mathrm{f}}$ reaches its maximum value $\lambda_{\mathrm{fm}}$. The failure envelopes are almost parallel to each other, and shift to the right as the concentration decreases. In other words, $\sigma_{\mathrm{f}}$ at a given strain becomes higher as the concentration increases. Furthermore, $\lambda_{\mathrm{fm}}$ increases with decreasing concentration. Both the modulus and $n(t)$ vary from point to point on a failure envelope. Therefore, the relationship between stress and extension ratio at any point on a failure envelope for an uncrosslinked polymer can be expressed by the following equations, described in a previous paper: ${ }^{2}$

$$
\sigma_{\mathrm{f}}=\frac{E\left(t_{\mathrm{f}}\right)}{3} \lambda_{\mathrm{f}}\left[\frac{n\left(t_{\mathrm{f}}\right)^{1 / 2}}{3} \mathscr{L}^{-1}\left(\frac{\lambda_{\mathrm{f}}}{n\left(t_{\mathrm{f}}\right)^{1 / 2}}\right)-\frac{1}{\lambda_{\mathrm{f}}^{2}}\right]
$$

In Figure 11, $\log \sigma_{\mathrm{f}} / E\left(t_{\mathrm{f}}\right)$ is plotted against $\log \left(\lambda_{f}-1\right)$ for two samples, LK and S50, as the representatives of all five samples, to avoid the complication of the figure. $E\left(t_{\mathrm{f}}\right)$ in this figure was evaluated from the isochronal stress-strain curve at $t_{\mathrm{f}}$. These modified failure envelopes show the concentration dependence. All the curves for different samples can be represented by a single straight line having the slope of -1 in the small $\left(\lambda_{\mathrm{f}}-1\right)$ region. However, in the large $\left(\lambda_{\mathrm{f}}-1\right)$ region, $\sigma_{\mathrm{f}} / E\left(t_{\mathrm{f}}\right)$ at a given $\left(\lambda_{\mathrm{f}}-1\right)$ becomes higher as the concentration increases. The higher the concentration, the steeper the large strain end of the envelopes becomes. This is different from our previous finding ${ }^{2}$ that all the curves for bulk polystyrenes having different molecular weights coincided well with each other over the entire range of $\left(\lambda_{f}-1\right)$ independent of molecular weight. The solid line in Figure 11 represents the values calculated from eq 14 employing the values of $n(t)$ given in Table II and Figure 7. As seen in Table II and Figure 7 , the $n(t)$ values employed are in good agreement with those obtained from the critical point

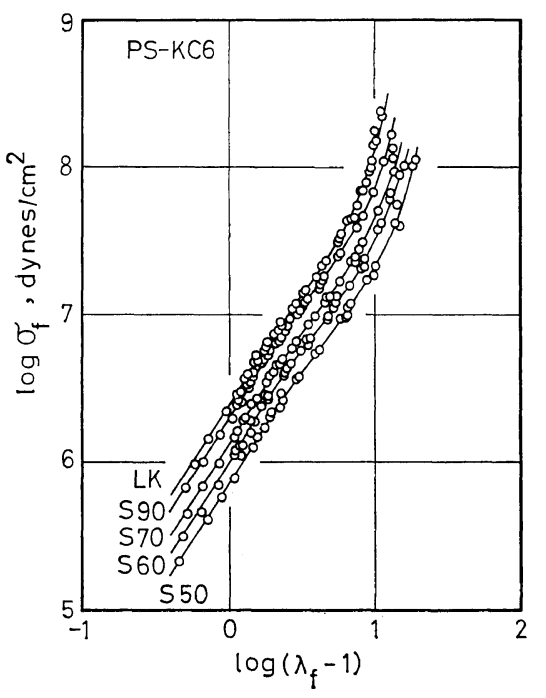

Figure 10. Failure envelopes for bulk polystyrene LK and its solutions in KC6.

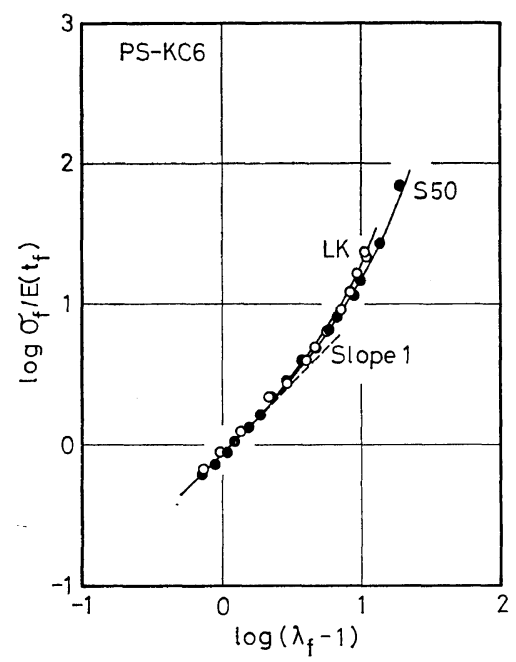

Figure 11. $\log \sigma_{\mathrm{f}} / E\left(t_{\mathrm{f}}\right) v s \cdot \log (\lambda-1)$ curves for bulk polystyrene LK and its solution S50. 
in Figure 9. That is, the concentration dependence of the modified failure envelopes in the large $\left(\lambda_{\mathrm{f}}-1\right)$ region is attributed to the fact that $M_{\mathrm{e}}$ depends upon concentration. The logarithmical plot of $\lambda_{\mathrm{fm}}$ against $\rho_{\mathrm{s}} w$ is also shown in Figure 7. The plot is approximated by a straight line whose slope is -0.9 . This slope is larger than that for the $n(t)^{1 / 2}$ plot in the same figure. However, the value of $\lambda_{\mathrm{fm}}$ is smaller than that of the corresponding $n\left(t_{\mathrm{f}}\right)^{1 / 2}$, indicating that $\lambda_{\mathrm{fm}}$ is not proportional to $n\left(t_{\mathrm{f}}\right)^{1 / 2}$, and that the failure occurs before the elongation in the specimen reaches its maximum extensibility. Furthermore, the ratio of $\lambda_{\mathrm{fm}}$ to $n\left(t_{\mathrm{f}}\right)^{1 / 2}$ becomes larger as the concentration decreases, indicating that the specimen can be elongated more easily as the viscous friction force decreases.

Time-Temperature Superposition Applied to the Fractural Properties

The failure stress $\sigma_{\mathrm{f}}$, failure strain $\gamma_{\mathrm{f}}$, breaking stress $\sigma_{\mathrm{b}}$, and strain at break $\gamma_{\mathrm{b}}$, as well as $n(t)^{1 / 2}$ and $E(t)$, measured as functions of extension rate or time at various temperatures can be superposed onto the respective master curves by horizontal shifts with the same shift factor for different quantities. The shift factors $a_{T}$ for all the samples are plotted against temperature in Figure 12. The reference temperature $T_{\mathrm{r}}$ is $103.7^{\circ} \mathrm{C}$ for all the samples. The temperature dependence of the shift factor shown in this figure can be expressed by the following WLFtype equation: ${ }^{18}$

$$
\log a_{T}=\frac{-C_{1}^{\mathrm{r}}\left(T-T_{\mathrm{r}}\right)}{C_{2}^{\mathrm{r}}+\left(T-T_{\mathrm{r}}\right)} .
$$

When an suitable reference temperature $T_{\mathrm{s}}$ is chosen for each sample, the shift factors for all the samples can be represented by the following original WLF equation: ${ }^{27}$

$$
\log a_{T}=\frac{-C_{1}\left(T-T_{\mathrm{s}}\right)}{C_{2}+\left(T-T_{\mathrm{s}}\right)},
$$

where $C_{1}=8.86$ and $C_{2}=101.6$.

In Figure 13 is shown the plot of $\log a_{T}$ against $\left(T-T_{\mathrm{s}}\right)$. The above equation means that when $\log a_{T}$ for all the samples is plotted against $\left(T-T_{\mathrm{s}}\right)$, a single curve can be obtained regardless of concentration. The reference temperatures $T_{\mathrm{r}}$ and $T_{\mathrm{s}}$ for the five samples are

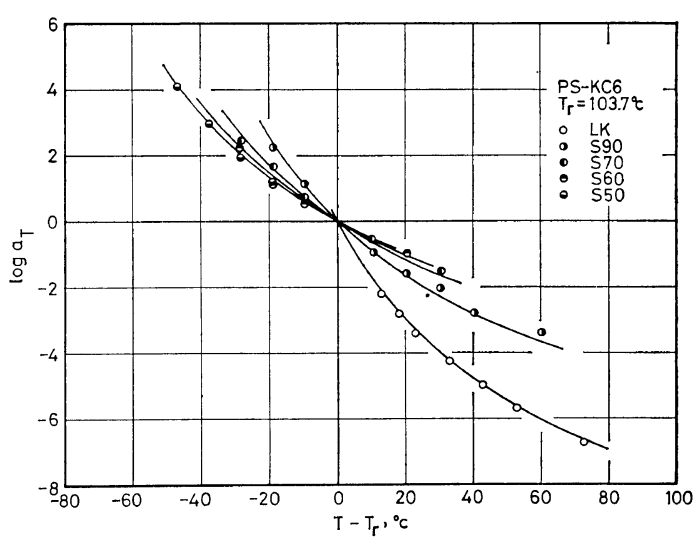

Figure 12. Temperature dependence of shift factor $a_{T}$ for bulk polystyrene LK and its solutions in KC6.

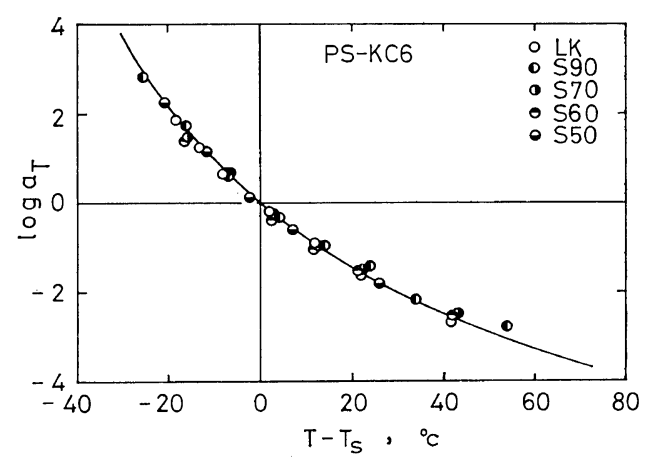

Figure 13. Logarithmic plot of $a_{T}$ against $\left(T-T_{\mathrm{S}}\right)$ for bulk polystyrene $\mathrm{LK}$ and its solutions in KC6.

Table III. Reference temperatures $T_{\mathrm{r}}$ and $T_{\mathrm{s}}$, the Vogel temperature $T_{0}$, and another temperature $T_{\mathrm{r}^{\prime}}$ at which all the solutions have the same fractional free volume of 0.0501 for bulk polystyrene $\mathrm{LK}$ and its solutions

\begin{tabular}{crrrr}
\hline Sample & $T_{\mathrm{r}},{ }^{\circ} \mathrm{C}$ & $T_{\mathrm{s}},{ }^{\circ} \mathrm{C}$ & $T_{0},{ }^{\circ} \mathrm{C}$ & $T_{\mathrm{r}}{ }^{\prime},{ }^{\circ} \mathrm{C}$ \\
\hline LK & 103.7 & 135.0 & 33.3 & 137.2 \\
S 90 & 103.7 & 110.4 & 8.7 & 112.6 \\
S 70 & 103.7 & 91.2 & -10.5 & 93.4 \\
S 60 & 103.7 & 82.4 & -19.3 & 84.6 \\
S 50 & 103.7 & 77.4 & -24.3 & 79.6 \\
\hline
\end{tabular}

tabulated in Table III. It is well-known that the constant $C_{1}$ and $C_{2}$ in the WLF equation can be related to the parameters in the free volume theory by the following equations: ${ }^{18,25}$ 


$$
C_{1}=B / 2.303 f_{\mathrm{s}},
$$

and

$$
C_{2}=f_{\mathrm{s}} / \alpha_{\mathrm{f}},
$$

where $B$ is a constant near unity, $f_{\mathrm{s}}$ the fractional free volume at $T_{\mathrm{s}}$, and $\alpha_{\mathrm{f}}$ the temperature coefficient of fractional free volume. Equation 17 is valid when the fractional free volume $f$ changes linearly with $\left(T-T_{0}\right)$, where $T_{0}$ is referred to as the Vogel temperature at which the fractional free volume becomes zero:

$$
f=\alpha_{\mathrm{f}}\left(T-T_{0}\right) \quad\left(T>T_{\mathrm{g}}\right)
$$

When all the shift factors obey the original WLF equation, the value of $\alpha_{\mathrm{f}}$ is equal to $4.82 \times 10^{-4}$ independent of concentration. The Vogel temperature $T_{0}$, and another temperature $T_{\mathrm{r}}{ }^{\prime}$ at which all the solutions have the same fractional free volume of 0.0501 , are also shown in Table III. $T_{\mathrm{r}}{ }^{\prime}$ is calculated from eq 16-18. For example, $T_{\mathrm{r}}{ }^{\prime}$ for the bulk sample $\mathrm{LK}$ is $137.2^{\circ} \mathrm{C}$. In the iso-free-volume state, all the solutions have the same monomeric friction factor. $T_{\mathrm{s}}, T_{0}$, and $T_{\mathrm{r}}{ }^{\prime}$ decrease with decreasing concentration. Similar results have been reported by several authors ${ }^{7,25}$ for the linear rheological properties of polymer solutions in the same concentration range.

\section{The Time Dependence of Failure and Breaking Properties}

As mentioned above, the rheological properties of polymer solutions depend not only upon molecular weight and concentration but also upon the segmental friction factor, which depends on concentration and temperature. Therefore, the concentration dependence of rheological properties of concentrated solutions must be discussed in the iso-free-volume state, where the monomeric friction factor is constant. ${ }^{7,18}$

In Figure 14 master curves of failure stress $\sigma_{\mathrm{f}}$ are shown for the bulk polymer LK and its solutions in the iso-free-volume state where $f=$ 0.0501 . The reference temperature $T_{\mathrm{r}}{ }^{\prime}$ for each sample is given in Table III. $\sigma_{\mathrm{f}}$ decreases with decreasing reduced rate of strain $\dot{\gamma} a_{T}$ and shows a plateau in the intermediate region because of high molecular weight $\mathrm{t}^{1,2}$ and concentration. The $\sigma_{\mathrm{f}}$ curves for the solutions intersect the curve for the bulk sample LK at $\dot{\gamma} a_{T}=0.02 \mathrm{sec}^{-1}$. The higher the strain rate and the lower the con- centration, the higher $\sigma_{\mathrm{f}}$ becomes at a given rate of strain. On the other hand, at lower strain rates, dilute solutions have lower $\sigma_{\mathrm{f}}$. The curves shift to the higher rate side as the concentration decreases. This dependence of $\sigma_{\mathrm{f}}$ on concentration is different from that on molecular weight for the bulk polystyrenes studied before. ${ }^{2}$ In the latter case, all the curves were parallel over the entire range of molecular weight without intersecting. This difference may be due to the fact that the ratio $\left(\lambda_{\mathrm{fm}} / n(t)^{1 / 2}\right)$ in eq 10 increases with decreasing concentration. The stress level in the high strain region is thus governed by the ratio $\left(\lambda_{\mathrm{fm}} / n(t)^{1 / 2}\right)$ rather than by $E(t)$.

The corresponding master curves of breaking stress $\sigma_{b}$ for the same samples in the same

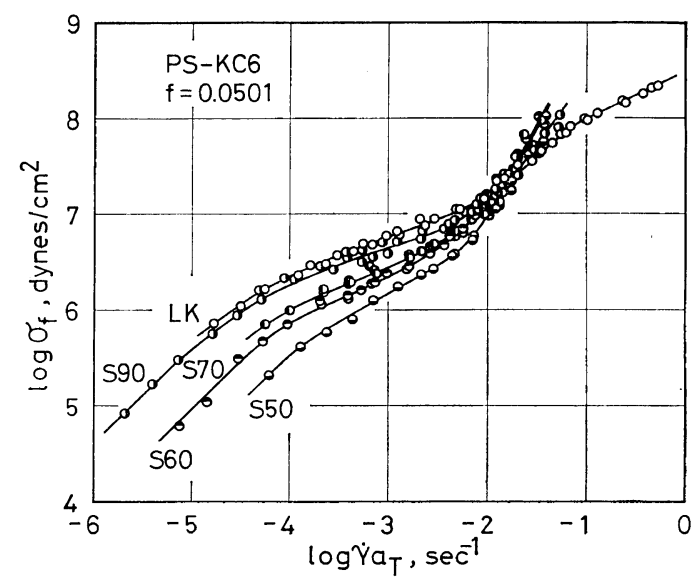

Figure 14. Master curves of $\sigma_{f}$ for bulk polystyrene LK and its solutions at the iso-free-volume state, $f=0.0501$.

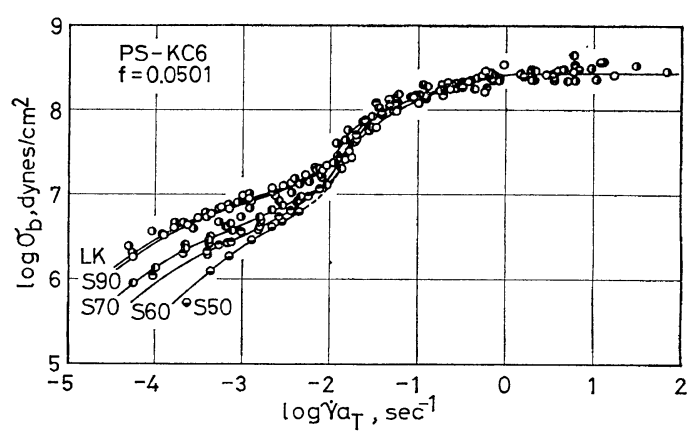

Figure 15. Master curves of $\sigma_{\mathrm{b}}$ for bulk polystyrene LK and its solutions at the iso-free-volume state, $f=0.0501$. 


\section{E. KAMEI and S. ONOGI}

iso-free-volume state are shown in Figure 15. The shape of the $\sigma_{\mathrm{b}}$ curves is very similar to that of the $\sigma_{\mathrm{f}}$ curves. It is clear that $\sigma_{\mathrm{b}}$ for all the samples is independent of concentration in the high rate region. In this region, $\sigma_{\mathrm{b}}$ is also independent of molecular weight, as described in a previous paper. ${ }^{2}$ The level of $\sigma_{\mathrm{b}}$ for bulk polystyrenes of different molecular weights is the same as that in this figure. In the high rate region, the test specimen breaks without showing a failure point. On the other hand, in the low rate region where the failure point begins to appear, the breaking stress depends upon concentration: the lower the rate of strain, the more remarkable the concentration dependence becomes. The concentration dependence of $\sigma_{\mathrm{b}}$ is very similar to the molecular weight dependence. ${ }^{2}$ The $\sigma_{\mathrm{b}}$ curves shift to the low rate side as concentration decreases, and the $\sigma_{\mathrm{b}}$ curves for all the samples at different concentrations show the plateau.
In Figure 16 master curves of $\gamma_{f}$ are shown for the same samples in the same iso-free-volume state. As is evident from this figure, the $\gamma_{f}$ curves are sigmoidal in shape and cross each other at $\dot{\gamma} a_{T}$ of about $0.02 \mathrm{sec}^{-1}$. Above this intersecting point, the higher the concentration, the lower the $\gamma_{f}$ at a given rate of strain becomes. On the other hand, below the intersecting point, the higher the concentration, the higher $\gamma_{f}$ at a given rate of strain becomes. This behavior is different from that of the molecular weight dependence of $\gamma_{\mathrm{f}}{ }^{2}$ in that all the curves are not parallel to each other.

The master curves of strain at break, $\gamma_{b}$, are shown in Figure 17. Each curve has a maximum and a minimum; $\gamma_{\mathrm{b}}$ depends upon concentration in the intermediate and low rate regions. The maxima for concentrated solutions are much sharper than that for the bulk polystyrene LK. Such a very sharp maximum is one of the prominent peculiarities of solutions. It seems

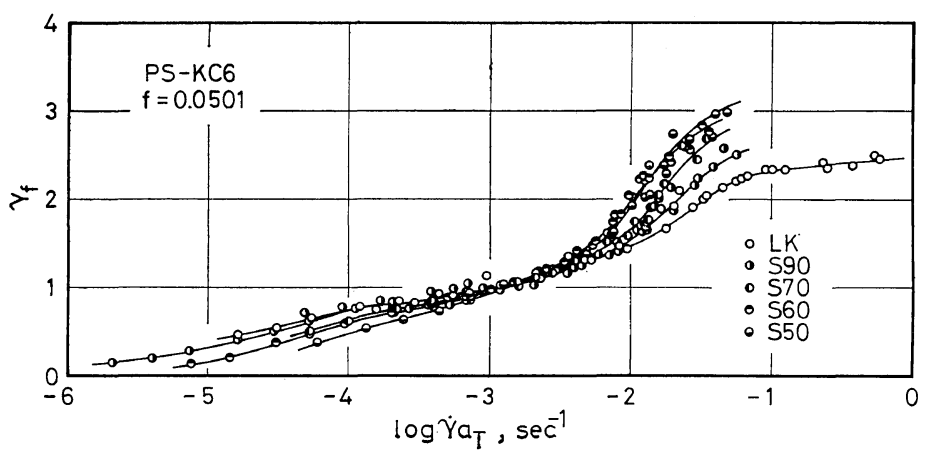

Figure 16. Master curves of $\gamma_{\mathrm{f}}$ for bulk polystyrene LK and its solutions at the iso-free-volume state, $f=0.0501$.

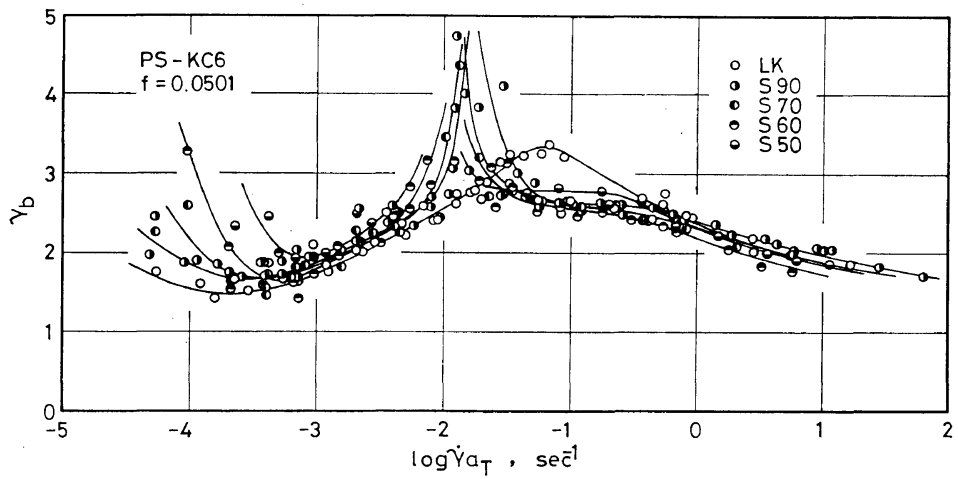

Figure 17. Master curves of $\gamma_{\mathrm{b}}$ for bulk polystyrene LK and its solutions at the iso-free-volume state, $f=0.0501$. 


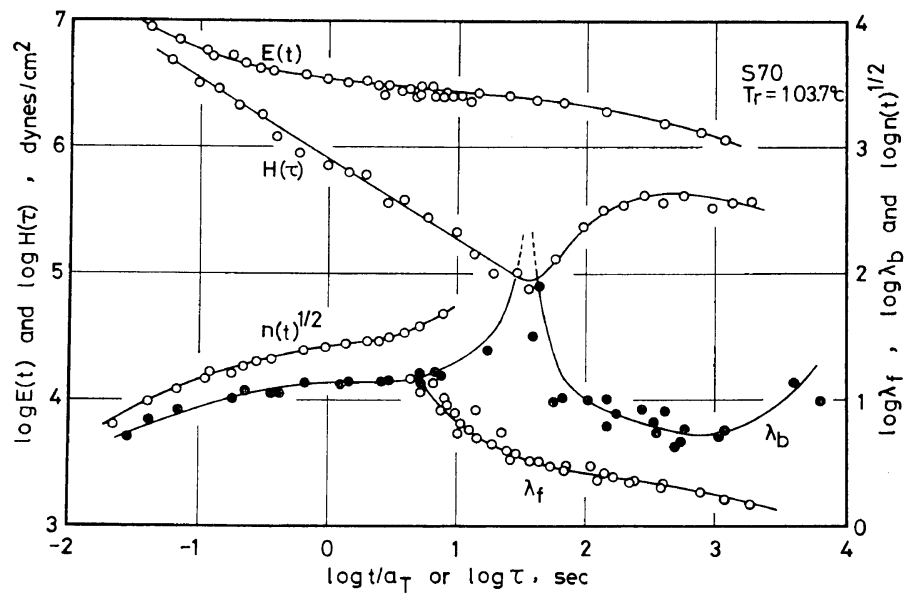

Figure 18. A comparison of $\lambda_{\mathrm{f}}$ and $\lambda_{\mathrm{b}}$ curves with master curves of $E(t), H(\tau)$, and $n(t)^{1 / 2}$ for $\mathrm{S} 70$ at a reference temperature of $103.7^{\circ} \mathrm{C}$.

that the rate of strain at which the maximum appears depends slightly upon concentration. The rate of strain at which the minimum appears also depends upon concentration. The rate of strain at the minimum point shifts to the left, and the minimum value of $\gamma_{b}$ becomes higher as the concentration decreases.

In Figure 18, the draw ratios at the failure point, $\lambda_{f}$, and at the breaking point, $\lambda_{b}$, for $S 70$ are shown along with other parameters evaluated from isochronal stress-strain curves, such as the elongational modulus $E(t)$, the time dependent maximum extensibility $n(t)^{1 / 2}$, and relaxation spectrum $H(\tau)$ determined from $E(t)$ by use of Tschoegl's equation. ${ }^{18}$ It is evident from this figure that we should consider two sets of relaxation times in this system. One set employs the Rouse type $\mathrm{e}^{18,28}$ relaxation times associated with cooperative motions of submolecules in backbone chains. The other is associated with slippage of entire molecular chains as a result of the release from entanglements. ${ }^{18}$ These two types of relaxation times might be distinguished conveniently by the minimum of the $H(\tau)$ curve. However, the two types of relaxation times would interlace in the region just below the minimum point of the relaxation spectrum $H(\tau)$. Therefore, the $\lambda_{\mathrm{f}}$ curve begins to appear earlier than the minimum point of $H(\tau)$, because the failure point can be considered to be the specific point at which the build-up of stress by chain extension counterbalances the reduction of stress by the slippage of molecular chains due to the destruction of structures formed by entanglements.

The position of the maximum point of the $\lambda_{b}$ curve coincides with that of the minimum point of $H(\tau)$. Furthermore, the $\lambda_{\mathrm{b}}$ curve looks like the image of the $H(\tau)$ curve. It is known from our previous study ${ }^{2}$ that the fracture of polymer melts at short time scales is not affected by chain slippage at all, suggesting that the breaking is governed mostly by the breaking of molecular chains themselves, which can move only on short range and in short time scales. In marked constant to this, the breaking in the longer time scale region, where the irrecoverable strain in the strained specimen cannot be ignored, ${ }^{2}$ is determined mostly by chain slippage.

The $n(t)^{1 / 2}$ curve is similar in its shape to the master curve of $\lambda_{b}$ in the short time scale region below the maximum point. However, $n(t)^{1 / 2}$ is higher than $\lambda_{b}$, suggesting that the breaking occurs before the maximum extensibility is attained. When $\lambda$ in eq 10 is equal to $n(t)^{1 / 2}$, the value of $\sigma(\lambda, t)$ becomes infinity. Therefore, the master curve of $n(t)^{1 / 2}$ implies the stored energy constant criterion for fracture. The master curve of $\lambda_{b}$ on the short time scale side below the maximum $\lambda_{b}$ may obey this criterion.

The extension of polymer chains between entanglement loci is controlled by the viscous 
frictional force. ${ }^{19-24}$ Therefore, the maximum extensibility of each coiled chain in the network structure depends upon time and temperature. A comparison of the $n(t)^{1 / 2}$ curve with the $H(\tau)$ curve suggests that deformation of the specimen obeys the molecular mechanisms which will be discussed below.

At low temperatures or high extension rates, each coiled chain in the structure furnishes a number of strands which pass through a plane perpendicular to the stretching direction and can support applied load. The materials as a whole responds as if the number of entanglements increased or the molecular weight between entanglement loci decreased. When the breaking occurs in this state, the strain at break becomes smaller than that at higher temperatures or lower extension rates. On the other hand, at times longer than the longest relaxation time of the Rouse type but still shorter than the shortest relaxation time for the molecular slippage process, the number of strands of each chain approaches unity. ${ }^{19-24}$ This means that the load is now supported by molecular chains acting as a whole. Then the time curve of $n(t)^{1 / 2}$ becomes almost flat, and $n(t)^{1 / 2}$ is proportional to $M_{\mathrm{e}}{ }^{1 / 2}$. When the time lengthens further, the slippage of molecular chains begins to occur. Then, the number of effective entanglements which can support the load decreases, and the molecular weight of molecular chains between entanglement loci apparently increases. Accordingly, $n(t)^{1 / 2}$ begins to increase again. As mentioned before, when cooperative motions of submolecules dominate, the scission of polymer chains is responsible for the fracture, even though there exists some slippage of molecular chains. When the molecular slippage is dominant, the fracture of a different type from that mentioned above takes place. Specimens broken above the maximum $\lambda_{b}$ are definitely different in their rupture surface and shape from those broken below the maximum. Below the maximum, the rupture occurs in a plane perpendicular to the stretching direction, and the fracture surface is quite smooth. However, above the maximum, the fracture surface is rough and the ruptured specimen is similar in shape to metals which have undergone ductile fracture. ${ }^{29}$ This type of rupture is caused by the slipping of chains which accompanies the breaking of entanglements.

\section{Consideration of the Failure Point}

The failure phenomenon at longer time scales mentioned above is associated with the onset of the breaking of entanglements and the slippage of polymer chains. Many investigations $\mathrm{s}^{30-33}$ of the nonlinear viscoelasticity of high polymers have shown that relaxation times corresponding to the relaxation of structures formed by entanglements of polymer chains depend upon the rate of strain, and that the dependence resembles the shear rate dependence of viscosity. Many constitutive equations ${ }^{34-37}$ hitherto proposed, in which this type of relaxation time is assumed to depend upon the rate of strain, can explain various kinds of nonlinear viscoelastic phenomena fairly well. As discussed in our previous paper, ${ }^{2}$ when the time required for a specimen to reach its failure point, $t_{\mathrm{f}}$, is assumed to be proportional to the characteristic time of relaxation at entanglement loci and is plotted logarithmically against the average rate of strain $\left\langle\Pi_{\Delta \mathrm{f}}^{1 / 2}\right\rangle$, the plot becomes similar to a plot of the shear viscosity against rate of shear. Furthermore, the slope of the linear part of the curve at higher rates is -0.818 . The limiting value of $t_{\mathrm{f}}$ at shorter rates, $t_{\mathrm{f} 0}$, is independent of rate and proportional to $M_{w}^{3.5}$, corresponding to the zero-shear viscosity. A key question is whether such a behavior can be observed also for concentrated polymer solutions or not.

In Figure $19, t_{\mathrm{f}}$ is logarithmically plotted against $\left\langle\Pi_{\Delta \mathrm{f}}^{1 / 2}\right\rangle$ for the five samples in the iso-freevolume state. The solid lines in this figure give theoretical curves obtained from Graessley's theory. ${ }^{4,5}$ Agreement between experimental and calculated values is excellent. However, the magnitude of $t_{\mathrm{f}}$ differs from sample to sample: the higher the concentration, the longer the value of $t_{\mathrm{f}}$ at the same rate of strain becomes. In order to clarify the concentration dependence of $t_{\mathrm{f} 0}$, it was plotted against $\rho_{\mathrm{s}} w$ in Figure 20. The $\mathrm{FLW}^{28}$ and Graessley ${ }^{38}$ theories predict that the characteristic relaxation time for entanglement is proportional to $\left(\rho_{\mathrm{S}} w\right)^{2.5}$ and $\left(\rho_{\mathrm{S}} w\right)^{1.5}$, respectively. Some rheological investigations ${ }^{10,15,18}$ on concentrated polymer solutions have shown that the characteristic time for entanglement is proportional to $\left(\rho_{\mathrm{s}} w\right)^{1.5} \sim\left(\rho_{\mathrm{s}} w\right)^{2.5}$. However, 


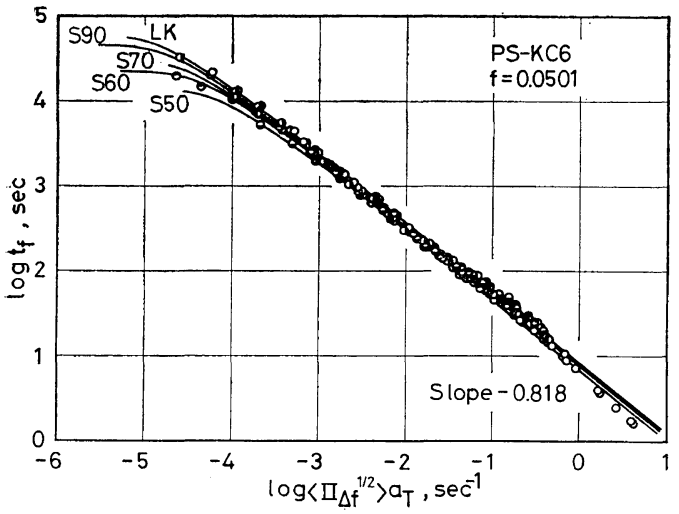

Figure 19. Logarithmic plot of $t_{\mathrm{f}}$ against average rate of strain for bulk polystyrene $\mathrm{LK}$ and its solutions at the iso-free-volume state, $f=0.0501$.

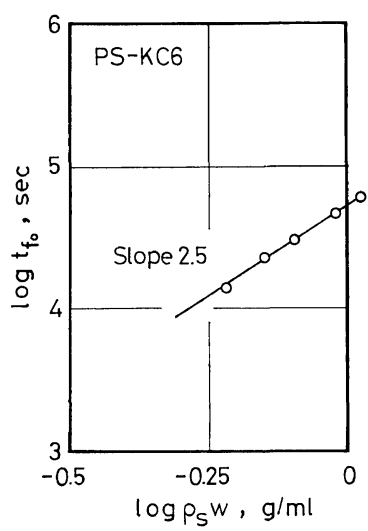

Figure 20. Concentration dependence of $t_{\mathrm{f} 0}$ for polystyrene solutions at the iso-free-volume state, $f=0.0501$.

there is no conclusive answer to this problem yet. The straight line in Figure 20 was drawn to have the slope of 2.5 and can represent experimental results satisfactorily. Thus, it follows that the failure point not only for bulk polystyrenes at elevated temperatures but also for concentrated solutions is closely associated with the characteristic relaxation time.

Finally, master curves of $t_{\mathrm{f}}$ and $t_{\mathrm{b}}$, which is the time required for the specimen to reach its breaking point, are plotted logarithmically against the average rate of strain for $\mathrm{S} 70$ at $T_{\mathrm{r}}=103.7^{\circ} \mathrm{C}$ in Figure 21. The $t_{\mathrm{f}}$ and $t_{\mathrm{b}}$ curves have the same slope of -0.818 in their intermediate portions. This result is consistent with the

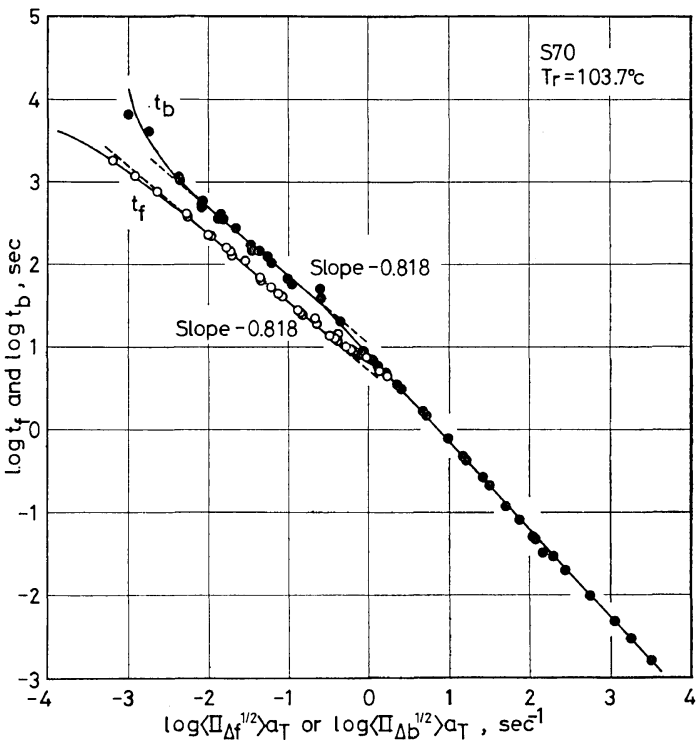

Figure 21. Master curves of $t_{\mathrm{f}}$ and $t_{\mathrm{b}}$ for $\mathrm{S} 70$ at a reference temperature of $103.7^{\circ} \mathrm{C}$.

previous findings for bulk polystyrenes. ${ }^{2}$ The failure and breaking behaviors can be also divided into the same three regions as in the case of the bulk polystyrenes:

Region 1: In this region, the breaking occurs below the failure point. This means that the breaking takes place after enough elastic energy to break polymer molecules is stored in these molecules or before the characteristic time is reached.

Region 2: In this region, both $t_{\mathrm{f}}$ and $t_{\mathrm{b}}$ curves are parallel to each other and have the same slope, namely -0.818 .

Region 3: In this region, $t_{\mathrm{f}}$ is constant and independent of rate of strain.

As can be seen in Figure 18, Region 1 is mainly governed by the relaxation time related to the motion of molecular chains between entanglement loci. It is considered that the breaking occurs mainly by the scission of such molecular chains between entanglement loci. Therefore, the fractural behavior is independent of concentration as well as molecular weight in this region, as can be seen from Figures 15 and 17. On the other hand, the fractural behavior in Regions 2 and 3 is attributed mainly to slippage of molecular chains which are released from entanglements. In this respect the fractural 
behavior is markedly different from that in Region 1 and is strongly dependent on polymer concentration, which determines the number and strength of entanglements.

\section{REFERENCES}

1. S. Onogi, T. Matsumoto, and E. Kamei, Polymer J., 3, 531 (1972).

2. E. Kamei and S. Onogi, Applied Polymer Symposia, No. 27, 19 (1975).

3. L. R. G. Treloar, "The Physics of Rubber Elasticity", Oxford University Press, London, 1958.

4. W. W. Graessley, J. Chem. Phys., 43, 2696 (1965).

5. W. W. Graessley, ibid., 47, 1942 (1967).

6. T. Masuda, N. Toda, Y. Aoto, and S. Onogi, Polymer J., 3, 315 (1972).

7. T. Masuda, Ph. D. Thesis, Kyoto University, Kyoto, 1973.

8. T. Masuda, Y. Ohota, M. Minamide, and S. Onogi, J. Soc. Material Sci., Japan 21, 436 (1972).

9. W. W. Graessley, R. L. Hazleton, and L. R. Lindeman, Trans. Soc. Rheol., 11, 267 (1967).

10. W. W. Graessley and L. Segal, Macromolecules. 2, 49 (1969).

11. L. A. Holmes and J. D. Ferry, J. Polym. Sci., Part-C, 23, 291 (1968).

12. S. Kusamizu, L. A. Holmes, A. A. Moore, and J. D. Ferry, Trans. Soc. Rheol., 12, 559 (1968).

13. Y. Einaga, K. Osaki, M. Kurata, and $\mathbf{M}$. Tamura, Macromolecules, 4, 87 (1971).

14. R. I. Wolkowicz and W. C. Forsman, ibid., 4, 184 (1971).

15. N. Nemoto, T. Ogawa, H. Odani, and $M$. Kurata, ibid., 5, 641 (1972).

16. T. Masuda, K. Kitagawa, T. Inoue, and S. Onogi, ibid., 3, 116 (1970).
17. D. C. Bogue, T. Masuda, Y. Einaga, and S. Onogi, Polymer J., 1, 563 (1970).

18. J. D. Ferry, "Viscoelastic Properties of Polymers", 2nd ed., John Wiley, New York, N.Y., 1970.

19. T. L. Smith and J. E. Frederic, J. Appl. Phys., 36, 2996 (1965).

20. T. L. Smith and R. A. Dickie, J. Polym. Sci., Part A-2, 7, 635 (1969).

21. J. C. Halpin, J. Appl. Phys., 36, 2975 (1965).

22. J. C. Halpin, J. Polym. Sci., Part-C, 16, 1073 (1967).

23. F. Bueche, J. Appl. Phys., 29, 1231 (1958).

24. F. Bueche, "Physical Properties of Polymers", Interscience, New York, N.Y., 1962.

25. G. C. Berry and T. G Fox, Advan. Polym. Sci., 5, 261 (1968).

26. S. Onogi, T. Masuda, and K. Kitagawa, Macromolecules, 3, 109 (1970).

27. M. L. Williams, R. F. Landel, and J. D. Ferry, J. Amer. Chem. Soc., 77, 3701 (1955).

28. J. D. Ferry, R. F. Landel, and M. L. Williams, J. Appl. Phys., 26, 359 (1955).

29. T. Yokobori, "Strength, Fracture, and Fatigue of Materials", Gihodo, Tokyo, 1955.

30. K. Osaki, M. Tamura, T. Kotaka, and M. Kurata, J. Phys. Chem., 69, 3642 (1965).

31. H. C. Booij, Rheol. Acta, 5, 215 (1966).

32. J. M. Simmons, J. Sci. Instr., 43, 887 (1966).

33. R. I. Tanner and J. M. Simmons, Chem. Eng. Sci., 22, 1803 (1967).

34. P. J. Carreau, Trans. Soc. Rheol., 16, 99 (1972).

35. D. C. Bogue, Ind. Eng. Chem. Fund., 5, 253 (1966).

36. M. Yamamoto, Trans. Soc. Rheol., 15, 331 (1971).

37. T. W. Spriggs, J. D. Huppler, and R. B. Bird, ibid., 10, 191 (1966).

38. W. W. Graessley, J. Chem. Phys., 54, 5143 (1971). 